\title{
An overview of rain attenuation research in Bangladesh
}

\author{
Md Abdus Samad $^{1}$, Md. Razu Ahmed ${ }^{2}$, Syed Zahidur Rashid ${ }^{3}$ \\ ${ }^{1}$ Department of Information and Communication Engineering, Chosun University, Gwangju 61452, Korea \\ ${ }^{1-3}$ Department of Electronics and Telecommunication Engineering, International Islamic University Chittagong, \\ Chattogram-4318, Bangladesh
}

\begin{tabular}{|c|c|}
\hline Article Info & ABSTRACT \\
\hline Article history: & \multirow{10}{*}{$\begin{array}{l}\text { The demands of shifting to the operating frequency of wireless telecommunication } \\
\text { systems at new higher frequency bands increase as day by day the necessity to transfer } \\
\text { more data volume through wireless networks. Bangladesh has launched its first satel- } \\
\text { lite, Bangabandhu-1, with } 40 \text { communication channels in the } C \text { and } K u \text { frequency } \\
\text { bands. Besides, a huge volume of terrestrial microwave backbone networks suffer } \\
\text { from fading during rain across the country. Bangladesh experiences heavy rainfall in } \\
\text { June-July-August. The rain has a remarkable impact on deteriorating the signal-to- } \\
\text { noise ratio at the receiver end. To implement the 5G network, 2.6- } 60 \mathrm{GHz} \text { frequency } \\
\text { bands are promising. However, the propagated waves in these bands are prone to fade } \\
\text { due to rain. Unfortunately, the rain attenuation model developed for other climatic } \\
\text { conditions can not be used readily without customization. In this regard, to maintain } \\
\text { quality telecommunication networks, proper rain attenuation model development is } \\
\text { crucial. This work reviews rain attenuation research in Bangladesh, global research } \\
\text { trends, and the research scope to manage rain attenuation. }\end{array}$} \\
\hline Received Apr 16, 2021 & \\
\hline Revised Jun 21, 2021 & \\
\hline Accepted Jun 24, 2021 & \\
\hline Keywords: & \\
\hline Bangabandhu-1 & \\
\hline Bangladesh & \\
\hline Microwave link & \\
\hline Rain attenuation & \\
\hline Rainfall rate & \\
\hline
\end{tabular}

This is an open access article under the CC BY-SA license.

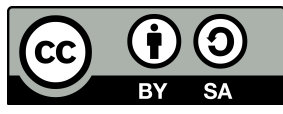

\section{Corresponding Author:}

Md Abdus Samad

Department of Electronics and Telecommunication Engineering

International Islamic University Chittagong

Chattogram-4318, Bangladesh

Email: masamad@iiuc.ac.bd

\section{INTRODUCTION}

The rain has a high impact on the propagation of electromagnetic waves. This force turns the attention of many researchers, organizations, and industry that results in plentiful studies worldwide to formulate the rain and its impact on rain attenuation with the propagated radio wave. These research works were conducted to inspect and estimate rain attenuation in different climatic areas, and over a wide range of frequency bands, especially for frequency bands over $10 \mathrm{GHz}$ [1]. In the literature, lots of such rain attenuation propagation model has been proposed.

Researchers are attempting to improvise existing models to fit local environmental conditions. A reasonable prediction of the amount of rain attenuation in a link is one of the keys to ensuring a communication link's quality of service. Assuming that the other communication elements are working correctly, the link availability can be guaranteed with proper rain attenuation modeling. On the other hand, through such estimation, it is possible to avoid overestimating the required transmission power that can be a reason for interference in the neighboring systems' radio frequency equipment.

The economy of Bangladesh is developing at a rapid pace. As per the UN's projection, Bangladesh will be graduated from the LDC country list in the next seven years. It is proven that, to progress in every 
spare of a nation, the accessibility to quality telecommunications plays a significant role. It has an influence on industrial-agricultural development, security, education, as well for the betterment of human life.

However, developing a quality telecommunication network at $5+\mathrm{GHz}$ frequency band is crucial to managing wave propagation in space from severe attenuation, mostly from rain in earth-space or terrestrial links. The current research works related to developing rain fade models and research scope for Bangladeshi climatic conditions have been investigated in this study. The main contributions of this paper are: i) the rainfall scenario and the climatic-dependent parameters that are related to the determination rain attenuation of Bangladesh are presented; ii) the carried research on rain attenuation about Bangladesh has been meticulously examined; iii) a generalized rain attenuation model, including all approaches of rain attenuation in Figure 1; and iv) there is a dedicated section focusing on the future research scope perspective of Bangladesh. The rest of the paper is arranged as follows: the impact of rainfall on radio wave propagation and rainfall scenario over Bangladeshi geographical location is given in section 2. Rain attenuation research outcomes in Bangladesh are presented in section 3, whereas the global tendency of rain attenuation modeling is presented in section 4 . In section 5, the Future research scope has been presented. Finally, section 6 ends with conclusive remarks.

\section{RAINFALL SCENARIO IN BANGLADESH}

\subsection{Impact of rain starts at $5+\mathbf{G H z}$}

The propagated wave of terrestrial and slant link communication gets attenuated due to rain at $10 \mathrm{GHz}$ in the temperate region. In [2], [3], some research suggests that such attenuation effects begin at 5-7 GHz. As a result, modeling rain attenuation is critical for propagating electromagnetic waves and achieving the necessary operation data transfer. Another atmospheric disturbance, like fog attenuation, dominates infrared and optical bands, while rain attenuation creates attenuation at $5+\mathrm{GHz}$. The rainfall diminishes system efficiency, and it necessitates deploying rain attenuation mitigation mechanisms for terrestrial and slant link communications systems at 5+ GHz. When the amount of rainfall, the operating frequency band, or the effective path length increases, rain attenuation mitigation becomes essential in such conditions. In tropical areas, the impact is extreme due to high precipitation rates and high rainfall intensity. The overall attenuation of the rain is directly related to the effective length of the link length.

The Rainfall is a substantial obstacle that hinders mm-wave propagation (30-300 $\mathrm{GHz}$ frequency) between the receiver and transmitter. The interaction between the rain and incident electromagnetic radio waves can limit propagated waves [4]. The attenuation drastically increases with increasing the rain rate. Such attenuation increases because the higher the rain rate means, the higher the raindrop increases the probability of creating a barrier to propagating radio waves. This barrier creates in the form of diffraction, scattering, absorption, as well as interaction. The increasing rain rate will intensify all events: diffraction, scattering, absorption, and interaction, which results in higher attenuation of the propagated wave that can make the communication link unreliable. Further, the propagated radio wave frequency is a significant parameter on which the amount of attenuation depends. In general, the higher the frequency higher the attenuation. Because the wavelength of higher frequency, the radio wave trends to be more comparable with the raindrop size, roughly $1.67 \mathrm{~mm}$ reported in [5]. The wavelength of the frequency 10 to $100 \mathrm{GHz}$ is 30 to $3 \mathrm{~mm}$, respectively. So, the wavelength of $100 \mathrm{GHz}$ frequency becomes more comparable with the raindrop size and attenuates more than the $10 \mathrm{GHz}$ frequency.

\subsection{Rainfall}

Bangladesh is a tropical country, and here happens heavy rain due to the Southern wind from the Bay of Bengal. The spatial distribution of typical rainfall in Bangladesh is shown in [6]. The distribution indicates that the highest rainfall occurs in the North-Eastern, Eastern, and Eastern parts. However, the rainfall distribution is not uniform over the country-the average annual rainfall varies from 2100 to $5100 \mathrm{~mm} / \mathrm{h}$ [7]. The low rainfall, less than $1500 \mathrm{~mm} / \mathrm{year}$, occurs in the Western part of Bangladesh [8]. The rainfall characteristics are significant in tropical regions compared with temperate areas. In [9], historical precipitation of rainfall data is available for 1951-2007. Rainfall is typically high in tropical regions, with large raindrop sizes and heavy thunderstorms throughout the year.

\subsection{Raindrop pattern}

In [10], tabulated results of raindrop size based on a few assumptions are available where different sizes of raindrops are mentioned, such as $2.5 \mathrm{~mm}, 0.5-3 \mathrm{~mm},<4 \mathrm{~mm}$, and $0.5-10 \mathrm{~mm}$. The raindrop shape that 
prevails here is the 'oblate spheroid' or 'spheres with the nose smashed' shape. On average, a raindrop size is between 0.1 to $5 \mathrm{~mm}$ was noticed here [10].

\section{RAIN ATTENUATION RESEARCH IN BANGLADESH}

Excellent insight into the rain attenuation scenario for Bangladesh about the satellite link with frequency $18.7 \mathrm{GHz}$ is reported in [11]. Due to beacon data unavailability, the authors mimic the same test done in Sparsholt, the United Kingdom, with just modifying the local temporal distribution of 1-minute rainfall rate (converted from yearly statistics) and making some corrections to "Sparsholt experiment." This study used the Rice-Holmberg model [12] to predict rain attenuation in Bangladesh. It was justified using the RiceHolmberg model over the ITU-R model from Sparsholt, United Kingdom, and Surabaya, Indonesia, experience (as Bangladesh also has a similar climate similar to Surabaya, Indonesia). According to this study, the rain fade in the slant direction is approximately $10 \mathrm{~dB}$ ( $K u$-band) and $30 \mathrm{~dB}$ ( $K a$-band), making it difficult to use these frequency bands in the subtropical zone.

The authors observed in [13] that rain fading is not crucial in the $C$-band but critical of the $K a$ and $K u$-bands. The authors have calculated that the rain fade from the suggested ITU-R rain intensity is significantly lower than the expected rainfall from converted results. In this study, the authors have used the Moupfouma model [14] with refined parameters to explain the 1-minute rain rate distribution in tropical areas. Using the Chebil rain rate conversion technique [15], the authors have calculated Bangladesh's rainfall rate for the South-East, South-West, the North-East, and the North-West region. In this study, the authors have not mentioned the boundaries of these regions.

In [16], the whole country was divided into four parts called South-East, South-West, North-East, and North-West, and rain attenuation was analyzed. In this study, the authors used vertically polarized signals and had calculated the rain fade for Singapore satellite ST1 located at $88^{\circ} \mathrm{E}$ longitude and earth station at Dhaka at $90^{\circ} \mathrm{E}$ longitudes and $24^{\circ} \mathrm{N}$ Latitude; the elevation angle is $61.80^{\circ}$. Their observation also reveals that the ITU-R rain attenuation model underestimates the actual rain attenuation, creating a significant error in designing a satellite-to-earth station's link budget and vice versa. The authors marked it challenging to design the link budget at $K u$ and $K a$-band in Bangladeshi climatic conditions based on the study of long-term statistical rainfall data. Their paper ended with the comment needed to measure raindrop size distribution and rain intensity to design a reliable microwave link in Bangladesh.

In [8], rain fade and intensity data and their effect on terrestrial and satellite microwave links' performance in Bangladeshi climate were investigated for both terrestrial and satellite microwave links. In this regard, long-term 13 years rainfall data from 34 meteorological stations of Bangladesh was used. In this study, predicted rain attenuation and calculated attenuation were determined through the ITU-R model at 4, 12, and $20 \mathrm{GHz}$ frequencies with vertical, horizontal, and circular polarization.

Rain attenuation due to the terrestrial microwave link in major cities such as Dhaka, Chittagong, Rajshahi, Sylhet, and Khulna and calculated average rain rate (0.01 percent) $119.7673,129.9933,109.1496$, 141.6991 and 114.6028 respectively [17]. The author justified his study on these five cities to argue that $80 \%$ of the devices in these cities need massive data to transfer through backbone networks. The author calculated the specific rain attenuation ITU-R P.838-1 [18] for horizontal and vertical polarization for a wide range of frequencies from 1 to $200 \mathrm{GHz}$. The outcome of this study showed that the ITU-R specified specific rain attenuation, and his calculated specific rain attenuation values produce different results. In the capital city Dhaka, this difference is maximum and is about $20 \%$ compared to the ITU-R specified value. The longer distance rain attenuation is comparatively less affected than, the shorter link length because of no- uniform rain distribution across the link. According to the observation, the horizontally polarized signal is more influenced by precipitation than the vertically and circularly polarized wave. Thus, using vertical polarization in heavy rain areas like Sylhet was expected to be a good choice.

In [19], rain attenuation all over Bangladesh was predicted through sectoring the whole country into eight parts for $K u$ and $K a$ bands. In this study, "Intelsat satellite 906 " positioned at $64.15^{\circ} \mathrm{E}$ on the Indian Ocean region satellite's beacon signals were used. In this work, to calculate the 1-min rain rate from yearlybased data, Chebil model [15] was used. "Contour map" of rain rate distribution above 0.01 percent of an average year at the $C$, and the $K u$ frequency band using ITU-R P.837-6 model [20] is reported in [21]. In this study, the rain rate from Bangladesh agricultural research council (BARC) and the meteorological department were used to develop the "contour map." 
It was predicted the rain attenuation for two probable earth stations of the Bangabandhu-1 satellite at Gazipur and Rangamati for vertical and horizontal polarization using ITU-R P.837-6 model [22]. In this work, the authors used 32 years of rainfall data from the Meteorological Department of Bangladesh. As the rainfall data was yearly, the authors used the Chebil model [15] to convert the annual rain rate to 1-minute rain rate data. According to their study, considering a $1 \%$ integration of time among the major cities of Bangladesh, the minimum rainfall rate is Isurdi $(108.406 \mathrm{~mm} / \mathrm{h})$ and Rajshahi $(108.980 \mathrm{~mm} / \mathrm{h})$, whereas the maximum rainfall rate is at Teknaf $(149.660 \mathrm{~mm} / \mathrm{h})$. The rainfall rate at the earth station of the Bangabandhu- 1 satellite is 110.500 $\mathrm{mm} / \mathrm{h}$ (Gazipur) and is $128.67 \mathrm{~mm} / \mathrm{h}$ (Rangamati). The authors have tabulated rain attenuation considering 1, $3,6,9,12$, and $14 \mathrm{GHz}$ frequencies vertical and horizontal in their works. In their works, there is no study of attenuation about uplink and downlink links. According to their results, the vertically polarized signal is better than horizontal because of raindrops' trends to be elliptical-shaped, which corrupt horizontal polarized signals more than vertical. Further, as the rainfall rate in Rangamati is higher than Gazipur by $18.17 \mathrm{~mm} / \mathrm{h}$, in both polarization cases, the Rangamati earth station suffers from more attenuation than Gazipur. Table 1 shows a summary of all contributions in Bangladesh. As discussed above, it is evident that there exists a lack of interest of researchers to address rain attenuation modeling in Bangladesh.

Table 1. Major research contribution to estimating rain attenuation in Bangladesh

\begin{tabular}{l} 
Ref. Contribution \\
\hline$[8]$ \\
$\begin{array}{l}\text { Investigated the rain fade and intensity data and their effect on the performance of terrestrial and satellite microwave } \\
\text { links in Bangladesh. }\end{array}$ \\
{$[11]$ Predicted earth-space link's rain attenuation using Rice-Holmberg model. } \\
Estimated rain fade using ITU-R recommended rain intensity noticed a considerably reduced value than that predicted \\
based on ITU-R dataset. \\
[17] Predicted rain attenuation due to the terrestrial microwave link in Dhaka, Chittagong, Rajshahi, Sylhet, and Khulna, and \\
calculated average rain rate. \\
Predicted rain attenuation all over Bangladesh by sectoring the whole country into eight parts for $K u$ and $K a$ bands. \\
[21] $\begin{array}{l}\text { Developed a "contour map" of rain rate distribution above } 0.01 \text { percent of an average year. } \\
\text { for vertical and horizontal polarization using ITU-R P.837-6 model [20]. }\end{array}$
\end{tabular}

\section{GLOBAL RESEARCH TRENDS}

The rain attenuation model can be considered as an empirical, physical-mathematical model, mathematical model, artificial intelligence-based model, machine learning-based model, fade slope model, and optimization-based model [23]. However, recently, the tendency of using an artificial neural network or deep learning-based rain attenuation models are proposed in the literature [24]. Thus merging the models in [23]-[26] a generalized rain attenuation model can be developed as shown in Figure 1 and this figure can be considered the "big-scenario" of the rain attenuation model to develop or apply the rain attenuation models. Here, the database section consists of the required parameters to develop the model. The parameter correction or generation section consists of several techniques that are used in the ordinary models, and these are effective rate determination technique, path length reduction technique, and rain height determination techniques which are generally used to determine the effective path length that exists in the rain for slant links. To work with the rain attenuation model, it needs long-term historical rain rate and rain attenuation datasets, or if the distribution of rain over a climatic zone is not available, then techniques such as "synthetic rain field" generation or "spatial distribution techniques" can help a lot. The necessary primary facts of these models are dependent on the accuracy level, i.e., how accurately these models can predict attenuation during a different time of the day and different seasons. Researchers are always in search of a better rain attenuation model with better accuracy. For example, the latest ITU-R model of rain attenuation is [27] that released in 2017 has already been examined and offered a modification with the help of the path length correction factor and full rainfall rate distribution. In recent years, the usage of artificial neural networks or highly computer intelligence technique-based techniques are noticed for fine-tuning of the rain attenuation propagation models [1], [28], [29]. Typically, deep learning or artificial neural network-based model considers lots of parameters, and through computation, it can predict rain attenuation. 


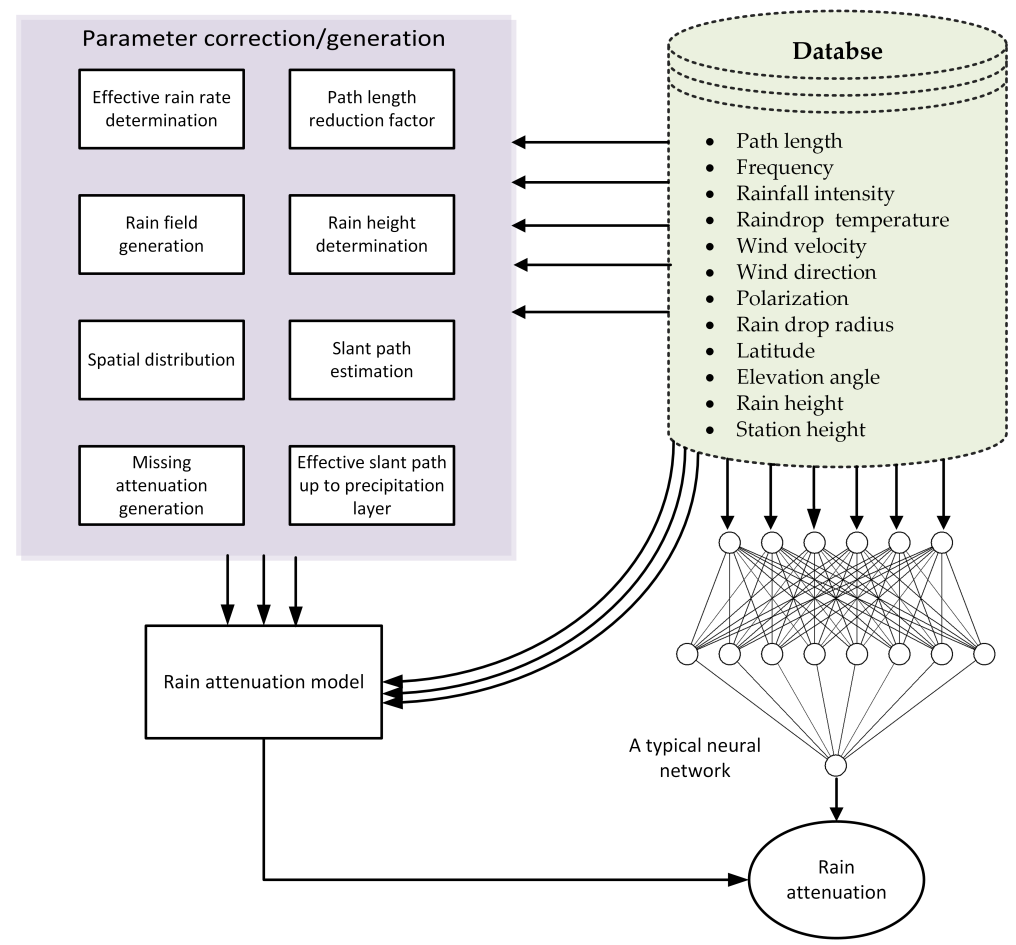

Figure 1. General procedure of the rain attenuation prediction model

\section{RESEARCH SCOPE IN BANGLADESH}

As said earlier, Bangladesh receives heavy rainfall due to the Southern winds from the Bay of Bengal. Along with wind flow in different seasons in diverse directions. According to the research results, both wind direction and velocity affect rain attenuation. For a proper estimation of rain attenuation, it is essential to study all factors (path length, frequency, rainfall intensity, raindrop temperature, humidity, pressure, wind velocity, wind direction, visibility, polarization, raindrop radius, percentage of time exceedance, latitude, elevation angle, rain height, azimuth, station height) in Bangladesh. However, the rain attenuation research outcome is minimal. Furthermore, it was tabulated the published research articles during January-July 2020, and it was recorded a total of 28 publications. Figure 2 shows the origination of the country of these papers. Although this study selected the paper for a seven-month duration, this short time also reflects the researchers' efforts to develop the counties' most rain attenuation model. For example, during the seven months Italy originated paper is three, and in the total enlisted rain attenuation model, Italy has seven contributions. So, it is evident that Bangladesh should also make research efforts to develop its "climate conditioned" rain attenuation model. In the next section, some important research issues are presented.

\subsection{Rain rate and attenuation measurement}

The rain mechanism is a spontaneous phenomenon with severe consequences for the propagation of high frequencies. The temporal and spatial inhomogeneity of rainfall can therefore increase the accessibility of the communication link. The distribution of rain cell size is essential for modeling the inclined path in radio communication [30]. With the rain gauge's assistance, the time series of rain is translated into the attenuation series, assuming a known value of the storm translation speed. The technique is known as the "synthesized storm technique," which includes [31] fine-resolution rain-rate time series as input. Therefore, to use synthetic techniques, real measurement of rain rate, rain cell measurement, and the storm velocity required to apply the "synthetic strom technique".

\subsection{Effective path length}

Earlier time, Bangladesh has to depend on other country's satellites to measure beacon signals for experimental purposes. It is an excellent opportunity for researchers to measure the rain height and the effective path length, especially at the $C$ and $K u$ bands, as the Bangabandhu satellite operates in this band of frequencies. 
Effective path length calculation procedure varies in slant link compared to the terrestrial link. For the terrestrial link, the effective path length depends mainly on rain distribution and rain type (stratiform and convective rain), whereas for the slant link, the effective path length up-to rainy link is defined by the rain cell structure. Hence, cloud height plays a vital role in determining the effective path length for slant links.

\subsection{Validate existing rain attenuation models}

Countries like Malaysia, Singapore, Brazil, Indonesia, South Korea, and Japan are experimenting with developing new rain attenuation models or modifying the existing model to fit their climatic conditions. For example, country-specific rain attenuation models are: Malaysia [4], Singapore [32], Brazil [33], and South Korea [34]. As Bangladesh also experiences lots of rain, it needs extensive research like the countries mentioned.

\subsection{Development of rain attenuation model for Bangladeshi climate}

Many countries have developed their rain attenuation model to fit the local climate. The authors have developed such a model by measuring directly long-term attenuation through a beacon signal [35]-[40]. Since now Bangladesh has its satellite, researchers can now measure the beacon signal using the Bangabandhu- 1 satellite with more manageable approval steps from the competent authority. Thus, through validating and remodeling of the existing models with the real measured data, a new rain attenuation prediction model for Bangladeshi climatic conditions may be possible.

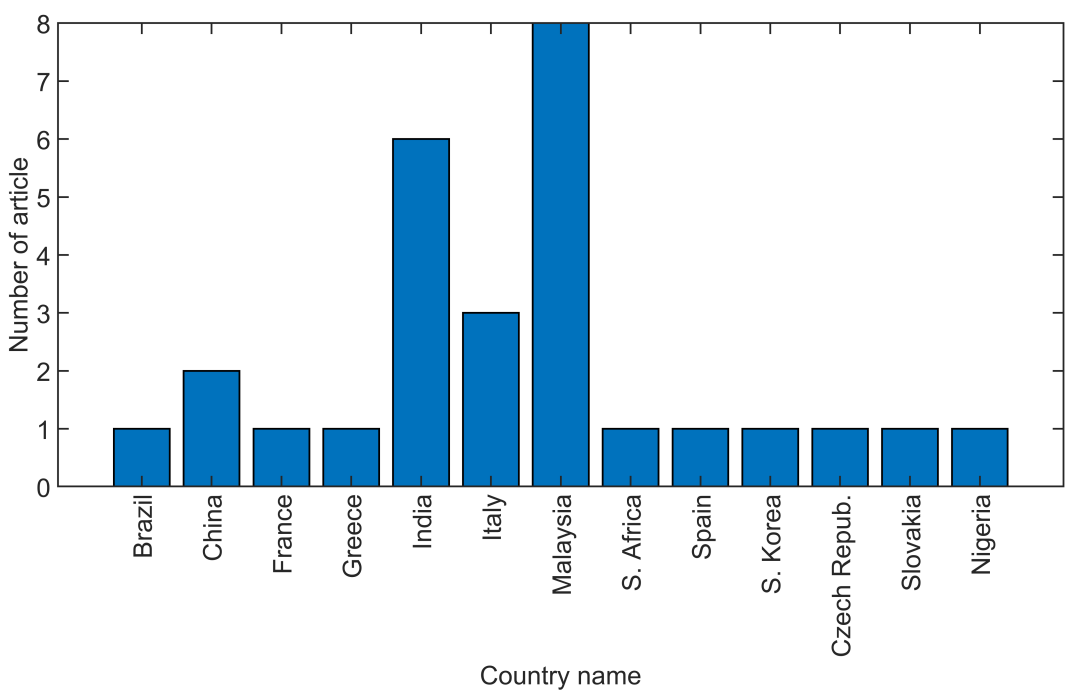

Figure 2. Comparison of the number of rain attenuation research outcomes during January-July 2020

\section{CONCLUSION}

Indeed, the temperate region model does not fit well in the tropical region, and researchers have made many modifications to the temperate region models to fit in tropical and subtropical countries like Malaysia, Singapore, Indonesia, India, and Brazil. Further, since the rain behavior in terms of rain rate, rain height is expected to be different in Bangladesh than in tropical and sub-tropical countries. Consequently, rain attenuation models developed in the tropical and subtropical regions can not be used directly without testing and verification for maintaining quality terrestrial and satellite radio links over Bangladesh. Thus for the betterment of $5 \mathrm{G}$ and beyond wireless telecommunication networks, it needs to pay attention to developing a rain attenuation model fitting in Bangladeshi climatic conditions.

\section{REFERENCES}

[1] A. D. Pinto-Mangones, J. M. Torres-Tovio, N. A. Pérez-García, L. A. da Silva Mello, A. F. Ruiz-Garcés, and J. LeónAcurio, "Improved ITU model for rainfall attenuation prediction of in terrestrial links," in Advances in Emerging 
Trends and Technologies, ICAETT 2019, Guayaquil, Ecuador: Springer, Mar 2019, vol. 1066, pp. 531-541, doi: 10.1007/978-3-030-32022-5_49.

[2] J. W. F. Goddard and M. Thurai, "Radar-derived path reduction factors for terrestrial systems,"in Tenth International Conference on Antennas and Propagation (Conf. Publ. No. 436), 1997, pp. 218-221, vol. 2, doi: 10.1049/cp:19970367.

[3] F. Moupfouma, "Improvement of a rain attenuation prediction method for terrestrial microwave links," in IEEE Transactions on Antennas and Propagation, vol. 32, no. 12, pp. 1368-1372, Dec. 1984, doi: 10.1109/TAP.1984.1143248.

[4] I. Shayea, T. A. Rahman, M. H. Azmi, and A. Arsad, "Rain attenuation of millimetre wave above $10 \mathrm{GHz}$ for terrestrial links in tropical regions," Transactions on Emerging Telecommunications Technologies, vol. 29, no. 8, p. e3450, Jul. 2018, doi: 10.1002/ett.3450.

[5] A. Tokay, P. G. Bashor, E. Habib, and T. Kasparis, "Raindrop size distribution measurements in tropical cyclones," Monthly Weather Review, vol. 136, no. 5, pp. 1669-1685, May 2008, doi: 10.1175/2007MWR2122.1.

[6] M. F. Ahmed, "Rainwater harvesting potentials in Bangladesh," in 25th WEDC Conference, Addis Ababa, Ethiopia: Loughborough University, 1999, pp. 363-365.

[7] B. M. A. Momin, "Rainfall in Bangladesh year: 2017 and 2018," Report, Bangladesh Water Development Board, 2019. [Online]. Available: https://www.bwdb.gov.bd/archive/pdf/6487.pdf

[8] M. Mahfujur Rashid, "Performance analysis of rain fades on terrestrial optical and microwave earth-to satellite links,” Ph.D. dissertation, Bangladesh University of Engineering and Technology, 2010.

[9] International Research Institute for Climate and Society, "Bangladesh historical precipitation monitoring." [Online]. Available: https://iridl.ldeo.columbia.edu/maproom/Agriculture/IFAD/Bangladesh_Precip.html (Accessed on Jun. 2, 2021)

[10] P. Biswas, M. Uddin, M. Islam, M. Sarkar, V. Desa, M. Khan, and A. Huq,"Harnessing raindrop energy in Bangladesh," in Proc. ICME'09, 2009, pp. 1-7. [Online]. Available: https://me.buet.ac.bd/icme/icme2009/ Proceedings/PDF/ICME09-AM-29.pdf

[11] S. Begum and I. E. Otung, "Characterization of rain attenuation in Bangladesh and application to satellite link design," Radio Science, vol. 43, no. 1, p. RS1008, Jan. 2008, doi: 10.1029/2007RS003634.

[12] P. Rice and N. Holmberg, "Cumulative time statistics of surface-point rainfall rates," IEEE Transactions on Communications, vol. 21, no. 10, pp. 1131-1136, October 1973, doi: 10.1109/TCOM.1973.1091546.

[13] M. R. Islam, M. A. Rahman, S. E. Hossain, and M. S. Azad, "Rain fade analysis on earth-space microwave link in a subtropical region," in 2008 International Conference on Electrical and Computer Engineering, 2008, pp. 793-798, doi: 10.1109/ICECE.2008.4769318.

[14] F. Moupfouma and L. Martin, "Modelling of the rainfall rate cumulative distribution for the design of satellite and terrestrial communication systems," International Journal of Satellite Communications, vol. 13, no. 2, pp. 105-115, Mar. 1995, doi: 10.1002/sat.4600130203.

[15] J. Chebil and T. Rahman, "Rain rate statistical conversion for the prediction of rain attenuation in Malaysia," Electronics Letters, vol. 35, no. 12, pp. 1019-1021, 1999, doi: 10.1049/el:19990685.

[16] M. M. Rashid, M. R. Islam, A. Z. Alam, S. Khan, O. O. Khalifa, and M. A. Rahman, "Rain fade and it's effect analysis for earth-to-satellite microwave link based on measured rainfall statistics in Bangladesh," 2009 IEEE 9th Malaysia International Conference on Communications (MICC), 2009, pp. 244-249, , doi: 10.1109/MICC.2009.5431505.

[17] S. Hossain, "Rain attenuation prediction for terrestrial microwave link in Bangladesh," Journal of Electrical and Electronics Engineering, vol. 7, no. 1, pp. 63-68, 2014. [Online]. Available: https://arxiv.org/abs/1406.5038

[18] ITU-R Recommendation, "P.838-1: Specific attenuation model for rain for use in prediction methods," ITU-R Recommendations, Report, 1999. [Online]. Available: https://www.itu.int/rec/R-REC-P.838

[19] M. Kamruzzaman and M. Islam, "Rain attenuation prediction for satellite communications link at Ku and Ka bands over Bangladesh," in 2014 International Conference on Electrical Engineering and Information and Communication Technology, 2014, pp. 1-6, doi: 10.1109/ICEEICT.2014.6919092.

[20] ITU-R Recommendation, "P. 837-6: Characteristics of precipitation for propagation modelling," ITU-R Recommendations, Report, 2017. [Online]. Available: https://www.itu.int/rec/R-REC-P.837/en

[21] A. Imran, M. T. Islam, A. Gafur, and Y. W. Rabby, "Rain attenuation prediction analysis and contour map design over Bangladesh," in 2015 18th International Conference on Computer and Information Technology (ICCIT), 2015, pp. 208-212, doi: 10.1109/ICCITechn.2015.7488069.

[22] M. I. Kamrul, A. Imran, A. Gafur, and S. Z. Rashid, "Rain attenuation estimation of vertical and horizontal polarizations for Bangabandhu-1 satellite," in 2018 International Conference on Advancement in Electrical and Electronic Engineering (ICAEEE), 2018, pp. 1-3, doi: 10.1109/ICAEEE.2018.8642972.

[23] M. A. Samad, F. D. Diba, and D.-Y. Choi, "A survey of rain attenuation prediction models for terrestrial links current research challenges and state-of-the-art," Sensors, vol. 21, no. 4, p. 1207, Feb. 2021, doi: 10.3390/s21041207.

[24] F. D. Diba, M. A. Samad, and D. Y. Choi, "The effects of rain on terrestrial links at $K, K a$ and $E$-bands in South Korea: Based on supervised learning," IEEE Access, vol. 9, pp. 9345-9355, 2021, doi: 10.1109/ACCESS.2021.3049825. 
[25] M. A. Samad and D.-Y. Choi, "Learning-assisted rain attenuation prediction models," Applied Sciences, vol. 10, no. 17, p. 6017, Aug. 2020, doi: 10.3390/app10176017.

[26] M. A. Samad, F. D. Diba, and D.-Y. Choi, "A survey of rain fade models for earth space telecommunication links taxonomy, methods, and comparative study," Remote Sensing, vol. 13, no. 10, p. 1965, May 2021, doi: $10.3390 / \mathrm{rs} 13101965$.

[27] International Telecommunication Union, "P.530-17: Propagation data and prediction methods required for the design of terrestrial line-of-sight systems," ITU-R Recommendations, Report, 2017. [Online]. Available: https://www.itu.int/rec/R-REC-P.530/en

[28] M. N. Ahuna, T. J. Afullo, and A. A. Alonge, "Rain attenuation prediction using artificial neural network for dynamic rain fade mitigation," in SAIEE Africa Research Journal, vol. 110, no. 1, pp. 11-18, March 2019, doi: 10.23919/SAIEE.2019.8643146.

[29] S. N. Livieratos and P. G. Cottis, "Rain attenuation along terrestrial millimeter wave links: A new prediction method based on supervised machine learning," in IEEE Access, vol. 7, pp. 138745-138756, 2019, doi: 10.1109/ACCESS.2019.2939498.

[30] N. H. H. Khamis, J. Din, and T. A. Rahman, "Determination of rain cell size distribution for microwave link design in Malaysia," in 2004 RF and Microwave Conference (IEEE Cat. No.04EX924), 2004, pp. 38-40, doi: 10.1109/RFM.2004.1411069.

[31] S. Begum, C. Nagaraja, and I. Otung, "Analysis of rain cell size distribution for application in site diversity," in 2006 First European Conference on Antennas and Propagation, 2006, pp. 1-5, doi: 10.1109/EUCAP.2006.4584777.

[32] J. Ong, Y. Choo, and S. Zhang, "Rain rate and attenuation prediction model for Singapore," in 1995 Ninth International Conference on Antennas and Propagation, ICAP '95 (Conf. Publ. No. 407), 1995, pp. 81-84 vol.2, doi: 10.1049/cp:19950388.

[33] M. Pontes, L. da Silva Mello, R. de Souza, and E. Miranda, "Review of rain attenuation studies in tropical and equatorial regions in Brazil," in 2005 5th International Conference on Information Communications and Signal Processing, 2005, pp. 1097-1101, doi: 10.1109/ICICS.2005.1689223.

[34] W. Kang, T. Kim, S. Park, I. Lee, and J. Pack, "Modeling of effective path-length based on rain cell statistics for total attenuation prediction in satellite link," in IEEE Communications Letters, vol. 22, no. 12, pp. 2483-2486, Dec. 2018, doi: 10.1109/LCOMM.2018.2873007.

[35] A. I. O. Yussuff, "Analysis of selected earth-space rain attenuation models for a tropical station," Indonesian Journal of Electrical Engineering and Computer Science (IJEECS), vol. 3, no. 2, pp. 383-391, 2016, doi: 10.11591/ijeecs.v3.i2.pp383-391.

[36] J. Yeo, Y. Lee, and J. Ong, "Ka-band satellite beacon attenuation and rain rate measurements in Singapore-comparison with ITU-R models," in 2009 IEEE Antennas and Propagation Society International Symposium, 2009, pp. 1-4, doi: 10.1109/APS.2009.5172073.

[37] I. F. Elshami, J. Din, L. H. Yin, and A. I. Elgayar, "Characterization of concurrent Ku band tropospheric scintillation and rain attenuation in Malaysia," Indonesian Journal of Electrical Engineering and Computer Science (IJEECS), vol. 15, no. 2, pp. 956-961, 2019, doi: 10.11591/ijeecs.v15.i2.pp956-961.

[38] C. J. Kikkert and O. P. Kenny, "A digital signal processing based Ka band satellite beacon receiver/radiometer," in 2008 2nd International Conference on Signal Processing and Communication Systems, 2008, pp. 1-8, doi: 10.1109/ICSPCS.2008.4813688.

[39] M. Tamrakar, K. Bandyopadhyay, and A. De, "Comparison of rain attenuation prediction models with Ku-band beacon measurement for satellite communication system," in 2010 International Conference on Signal Processing and Communications (SPCOM), 2010, pp. 1-5, doi: 10.1109/SPCOM.2010.5560497.

[40] S. Yokozawa, M. Kamei, and H. Sujikai, "Rain attenuation characteristics for 21-GHz-band satellite broadcasting measured by beacon signal," in 2019 International Symposium on Antennas and Propagation (ISAP), 2019, pp. 1-3. 